\title{
Overexpression of bone morphogenetic protein 4 in STO fibroblast feeder cells represses the proliferation of mouse embryonic stem cells in vitro
}

\author{
Gu-Hee Kim ${ }^{1}$, Gong-Rak Lee ${ }^{2}$, Hyung-Im Choi ${ }^{1}$, \\ Neung-Hwa Park ${ }^{3}$, Hun Taeg Chung ${ }^{1}$ \\ and In-Seob Han ${ }^{1,4}$ \\ ${ }^{1}$ School of Biological Sciences \\ ${ }^{2}$ Department of Medical Science \\ School of Medicine \\ University of Ulsan \\ Ulsan 680-749, Korea \\ ${ }^{3}$ Department of Internal Medicine \\ Ulsan University Hospital and School of Medicine \\ University of Ulsan \\ Ulsan 680-749, Korea \\ ${ }^{4}$ Corresponding author: Tel, 82-52-259-2352; \\ Fax, 82-52-259-1694; E-mail, hanis @ulsan.ac.kr \\ http://dx.doi.org/10.3858/emm.2012.44.7.052
}

Accepted 10 May 2012

Available Online 17 May 2012

Abbreviations: BMP, bone morphogenetic protein; ESC, embryonic stem cell; rAd-dnlkB, recombinant adenovirus-dominant negative IאB

\begin{abstract}
Embryonic stem cells (ESCs) can be propagated in vitro on feeder layers of mouse STO fibroblast cells. The STO cells secrete several cytokines that are essential for ESCs to maintain their undifferentiated state. In this study, we found significant growth inhibition of mouse ESCs (mESCs) cultured on STO cells infected with adenovirus containing a dominant-negative mutant form of $I_{\kappa} B\left(r A d-d n l_{\kappa} B\right)$. This blockage of the NF- $k B$ signal pathway in STO cells led to a significant decrease in $\left[{ }^{3} \mathrm{H}\right]$ thymidine incorporation and colony formation of mESCs. Expression profile of cytokines secreted from the STO cells revealed an increase in the bone morphogenetic protein4 (BMP4) transcript level in the STO cells infected with adenoviral vector encoding dominant negative $I_{\kappa} B\left(r A d-d n I_{\kappa} B\right)$. These results suggested that the NF- $\kappa B$ signaling pathway represses ex-
\end{abstract}

pression of BMP4 in STO feeder cells. Conditioned medium from the rAd-dnlkB-infected STO cells also significantly reduced the colony size of $\mathrm{mESCs}$. Addition of BMP4 prevented colony formation of mESCs cultured in the conditioned medium. Our finding suggested that an excess of BMP4 in the conditioned medium also inhibits proliferation of $\mathrm{mESCs}$.

Keywords: bone morphogenetic protein 4; culture media, conditioned; embryonic stem cells; feeder cells; NF-кB

\section{Introduction}

Several growth factors that are required for selfrenewal of embryonic stem cells (ESCs) are usually provided either exogenously or by feeder cells (Smith, 2001; Ahn et al., 2010). Different embryonic fibroblast cell types have been tested as feeder cells for culture of undifferentiated ESCs. These cells secrete several cytokines that are essential for maintaining mouse ESCs (mESCs) in an undifferentiated state. STO, a transformed mouse fibroblast line, is commonly used as feeder cells to support mESC growth (Smith et al., 1988). mESCs depend on bone morphogenetic protein (BMP) and leukemia inhibitory factor (LIF) to maintain their pluripotent status (Brons et al., 2007). Conditioned medium from STO cell culture has been studied as an economical alternative to these cytokines commonly used in mESC growth media.

BMPs are members of the transforming growth factor beta (TGF- $\beta$ ) family and have been implicated in embryonic development, including bone formation and repair, organogenesis, pattern formation in the early embryo, and epithelial-mesenchymal interactions (Jones et al., 1991; Vainio et al., 1993; Fainsod et al., 1994; De Robertis and Sasai, 1996). BMP4 is required at a very early step as an important regulator of the growth of hematopoietic stem cells (HSC), participating in the control of their proliferation, expansion and differentiation (Pearson et al., 2008). The major effect of BMP4 on the self-renewal of 
ESCs is accomplished by means of the inhibition of both extracellular receptor kinase (ERK) and p38 mitogen-activated protein kinase (MAPK) pathways (Qi et al., 2004). Human BMP4 and hematopoietic cytokines have been shown to modulate the proliferative and differentiative potentials of definitive fetal, neonatal, and adult hematopoietic progenitors (Bhatia et al., 1997, 1999; Bhardwaj et al., 2001; Murdoch et al., 2002). ESCs cultured on embryonic feeder cells can be induced into trophoblastic differentiation by collagen IV or BMP4 (Xu et al., 2002; Schenke-Layland et al., 2007). Early exposure to BMP4 inhibits the neurogenic differentiation of ESCs, whereas later exposure causes induction of peripheral neuronal differentiation (Schulz et al., 2004). These studies indicate that BMP4 is required for self-renewal of ESCs or for differentiation into a specific lineage if they are provided with the correct cues. BMP4 regulation is complex and precisely controlled by various transcription factors and interand intra- cellular signaling pathways. A recent report showed that TNF- $\alpha$ represses transcription of human BMP4 in lung epithelial cells through the nuclear factor $\kappa \mathrm{B}(\mathrm{NF}-\kappa \mathrm{B})$ signaling pathway (Zhu et al., 2007 ). Sequence analysis has suggested that the 5 ' upstream region of BMP4 contains a putative NF- $\kappa B$ binding site. However, few functional results are currently available regarding the regulation of BMP4 gene expression in any organ.

Here we blocked the NF- $\mathrm{B}$ B signaling pathway in STO feeder cells by infection with recombinant adenovirus encoding a dominant-negative mutant of $I_{\kappa} B\left(r A d-\left.d n\right|_{\kappa} B\right)$. As shown in lung epithelial cells (Zhu et al., 2007), this blockade induced high expression of BMP4 in the STO cells and consequently resulted in a decreased survival of mouse ESCs in co-cultures. The addition of exogenous BMP4 to ES culture medium also inhibited colony formation from mESCs. These findings suggest that the regulated expression of BMP4 in feeder cells is important for self-renewal of mESCs in vitro.

\section{Results}

\section{Inhibition of the NF- $\kappa$ B signaling pathway in STO cells decreases proliferation of mESCs}

To investigate the cellular and molecular consequences of $I_{\kappa} B$ in STO cells, we constructed an adenovirus plasmid expressing a dominant-negative mutant of $I_{\kappa} B\left(r A d-d n I_{\kappa} B\right.$ ) that cannot be phosphorylated and thus abrogates the NF- $\mathrm{KB}$ signaling pathway and a negative control plasmid expressing EGFP (rAd-GFP). We infected STO feeder cells by incubation with $r A d-\left.d n\right|_{\kappa} B$ virus at a multiplicity of infection (MOI) values of 3, 5, and 10 and then used these feeder cells for culture of mESCs. Infection of STO cells by the rAd-dnl $\kappa B$ reduced the number and colony size of co-cultured mES cells as $\mathrm{MOI}$ increased, while the number and colony size of control STO cells infected by rAd-GFP was unaffected (Figure 1). These data suggest that inhibition of the $\mathrm{NF}-\kappa \mathrm{B}$ signaling pathway in STO cells prevents growth of mESCs. Using reverse transcription (RT)-PCR analysis with $r A d-d n l \kappa B$ specific primers, we confirmed that the reduced proliferation was caused by STO cells but not by mESCs themselves (Supplemental Data Figure S1).

To investigate whether inhibition of the NF- $\kappa B$ pathway in STO cells affected proliferation of mESCs, we performed a ${ }^{3} \mathrm{H}$-thymidine incorporation assay. The STO cells expressing dnl $\mathrm{K} B$ markedly inhibited cellular proliferation of mESCs at $24 \mathrm{~h}, 48 \mathrm{~h}$, and $72 \mathrm{~h}$ (Figure 2A). These data indicated that the

A

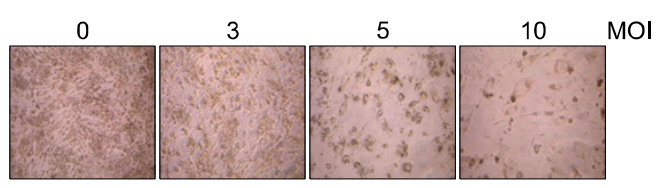

B

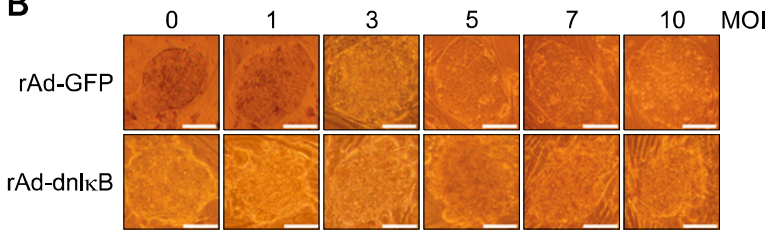

C
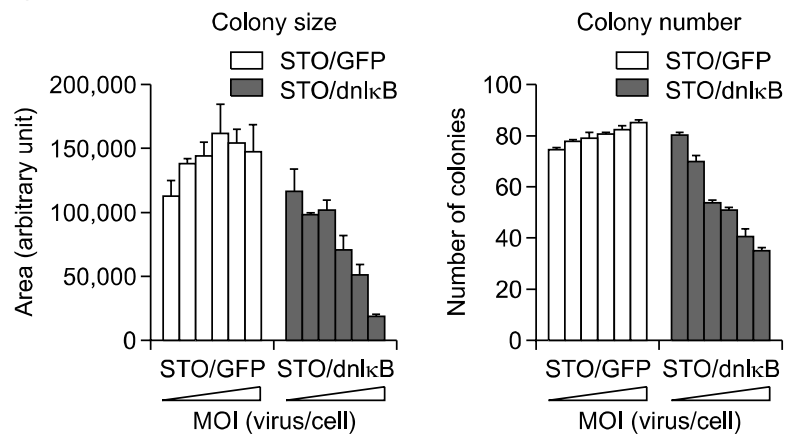

Figure 1. Inhibition of NF- $\mathrm{KB}$ signaling in STO feeder cells by infection with $\mathrm{rAd}-\mathrm{dn} \mid \kappa \mathrm{K}$ prevents colony formation of mESCs. Photographs of representative colonies were taken on day 4 of treatment. (A) Morphological changes of mESCs cultured on STO cells infected by rAd-dnl $\kappa B$ at the indicated MOI. (B) The morphology of mESC colonies cultured on STO feeder cells. (C) The number and size of colonies of mESCs cultured on STO cells infected by rAd-dnlkB. The colony number was counted under a microscope, and the colony size was determined by measuring the colony area with the Image $\mathrm{J}$ program $(\mathrm{NIH})$. All assays were performed in duplicate (scale bar, $50 \mu \mathrm{m}$ ). 
A

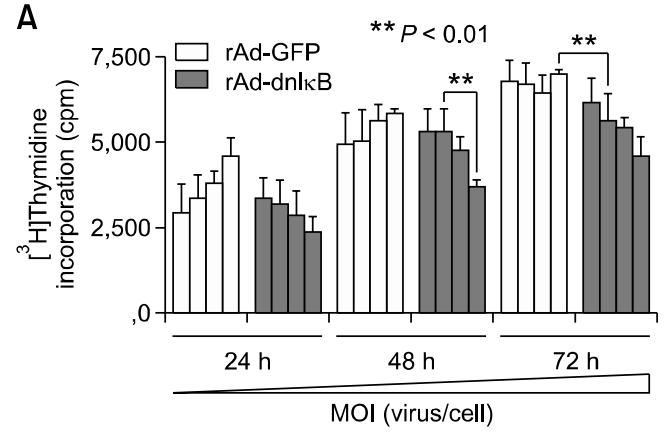

B

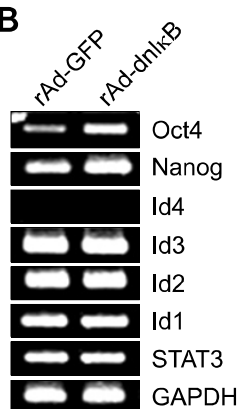

C

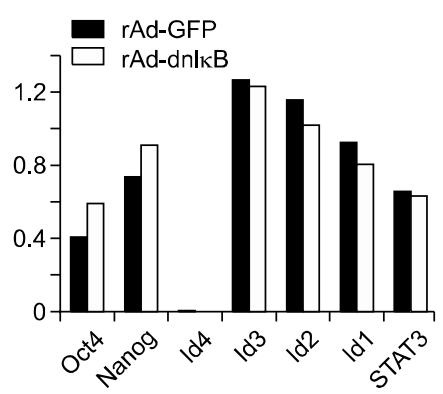

Figure 2. Proliferation of mES cells was inhibited by the STO cells infected with rAd-dnIKB. (A) The mESCs were cultured on STO cells as indicated, and proliferation of mESCs was measured by $\left[{ }^{3} \mathrm{H}\right]$ thymidine incorporation assay. (B) RT-PCR analysis of the ESCs marker genes. GAPDH mRNA was measured as a control. (C) The relative density of gene expression was determined by dividing the density of each gene by that of GAPDH. ${ }^{* *}$ A statistically significant difference $(P<0.01)$ compared to the rAd-GFP control.

NF-kB signaling pathway was essential for STO cells to function as feeder cells in inducing selfrenewal of $\mathrm{mES}$ cells.

We analyzed transcription of proliferation-related genes of ESCs when these cells were co-cultured with the STO cells infected by rAd-dnlкB. Figure 2B showed that mRNA levels of Oct 4 and Nanog were increased in the ESCs, whereas the expression levels of $I d$ genes were unchanged.

\section{Conditioned medium from the STO cells infected by rAd-dnlKB reduces the colony size of $\mathrm{mESCs}$}

To investigate whether inhibition of the $N F_{\kappa B}$ signaling pathway destroyed the characteristic of STO cells to secrete ESC proliferation-stimulating factors, we analyzed colony formation of mESCs after culture with the conditioned medium collected from the rAd-dnlkB-infected STO cell cultures (Figure 3 ). The colony size decreased significantly as conditioned medium from rAd-dnlkB-infected STO cells was increased but remained unchanged in control medium from rAd-GFP-infected STO cells. This suggests that STO cells secrete a factor which

A

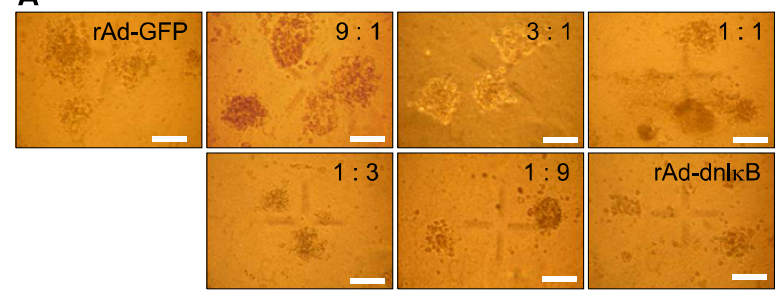

B

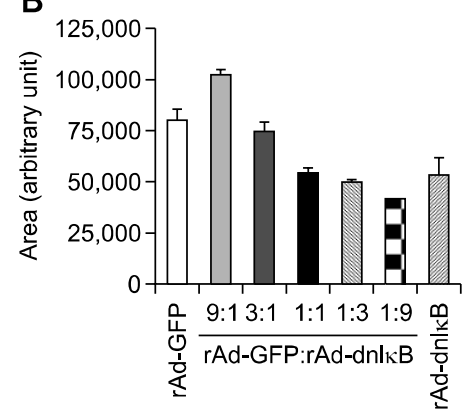

Figure 3. Effect of the conditioned media from STO cells infected with rAd-dn|KB. Conditioned medium from STO cells infected by rAd-C was mixed with that of STO cells infected by rAd-GFP at the indicated ratio (rAd-GFP only, 9:1, 3:1, 1:1, 1:3, 1:9, and rAd-dnlкB only). ESCs were cultured in the conditioned medium for three days. (A) Morphology of representative colonies and $(B)$ comparison of colony sizes of mESCs. The colony size ( $>10$ colonies/sample) was determined by measuring the colony area with the Image J program $(\mathrm{NIH})$. All assays were performed in duplicate (scale bar, $25 \mu \mathrm{m}$ ).

stimulates proliferation of mESCs mediated by the NFKB signaling pathway.

\section{Inhibition of the NFKB signal transduction pathway increases BMP4 expression in STO feeder cells}

To identify factor which changed upon inhibition of the NFKB signaling pathway in STO cells, we analyzed the mRNA levels of BMP4, LIF, and Wnts genes because they are known as critical factors for maintaining the characteristics of mESCs (Hao et al., 2006). RT-PCR analysis detected higher expression of BMP4 in the STO cells infected by rAd-dnlkB, whereas mRNA levels of LIF and Wnts genes were not significantly different (Figure 4A). Real-time PCR confirmed level of BMP4 mRNA was increased in the $\mathrm{rAd}-\mathrm{dn} / \kappa \mathrm{K}$-infected STO cells (Figure 4B).

These data suggest that the enhanced level of BMP4 in STO feeder cells might inhibit the proliferation of mESCs. 
A

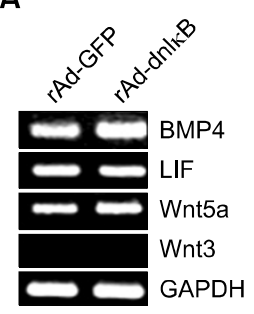

B

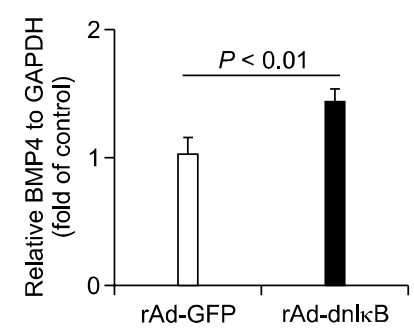

C

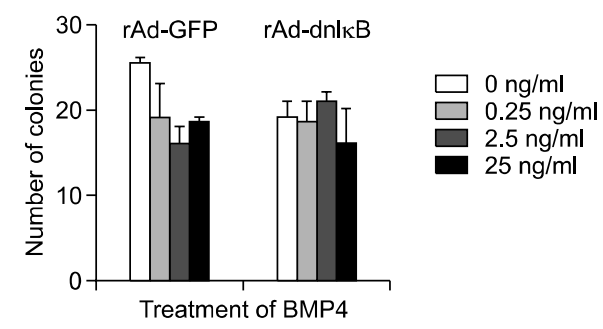

D

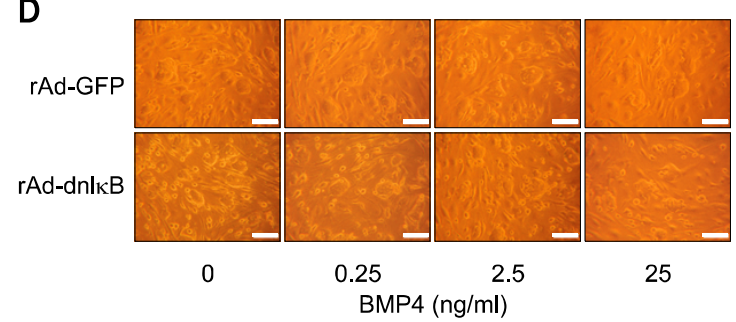

Figure 4. Overexpression of Bmp4 in STO cells infected with rAd-dnlкB inhibits mESC proliferation. (A) RT-PCR analysis of the STO feeder cells infected by rAd-GFP and rAd-dnl $/ \mathrm{B}$. The rAd-dnl $\mathrm{KB}$ infection induced overexpression of Bmp4 gene in the STO cells. GAPDH mRNA was measured as a control. (B) The relative density of genes expression was determined by dividing the density of each gene by that of GAPDH. The level of BMP4 mRNA was analyzed by using real time PCR. Results are representative of three independent experiments. (C) The number of colonies of mESCs was counted after culturing in the conditioned medium produced from STO cells plus exogenous BMP4. Addition of BMP4 resulted in a decrease in the number of colonies produced from the conditioned medium of rAd-GFP medium compared to that of $\mathrm{rAd}-\mathrm{dn} \mid \kappa B$ medium. (D) Morphology of mESC colonies cultured in the conditioned medium plus exogenous BMP4.

\section{Over-production of BMP4 in STO feeder cells leads to decreased $\mathrm{mESC}$ proliferation}

To confirm whether over-production of BMP-4 led to inhibition of $m E S C$ proliferation, we added recombinant BMP4 to the mESC culture medium. Addition of exogenous BMP4 $(2.5-25 \mathrm{ng} / \mathrm{ml})$ reduced the colony number of mESCs co-cultured with rAd-GFP-infected STO feeder cells $(P<0.01)$ to a similar level as in non-treated mESCs co-cultured with the rAd-dnIкBinfected STO cells (Figures 4C and 4D). These results indicate that BMP4 is required in appropriate amounts to maintain $\mathrm{mESC}$ self-renewal.

\section{Discussion}

Mammalian feeder cells continue to be broadly accepted as the method for maintaining ESC culture because these cells seem to produce some unidentified factor that makes them very effective. Extensive cell proliferation must be carefully addressed in stem cell research before theoretical possibilities of stem cells are translated into clinical applications (Park et al., 2008). One active area of current stem cell research includes identification of the molecular processes underlying the uniform maintenance of proliferation, the undifferentiated state and pluripotency. Defining some of the signals important in the self-renewal of human ESCs is necessary to eliminate the need to include animal-derived materials. BMP4 has been identified as a good candidate for extensive ex vivo production of hESCs for therapeutic applications (Bhatia et al., 1999; Hollnagel et al., 1999; Ying et al., 2003; Vicente Lopez et al., 2011). Suppression of BMP signaling has been beneficial and not deleterious to hESC culture (Xu et al., 2005). In contrast, BMP4 was not responsible for the activity of STO cells that support ES self-renewal or proliferation (Hao et al., 2006). Here we more closely evaluated the ability of BMP-4 from feeder cells to maintain mESC culture. We found that a dominant-negative catalytically inactive construct ( $\mathrm{rAd}-\mathrm{dn} \mid \kappa \mathrm{B})$ led to profound attenuation of NF- $\mathrm{KB}$ signaling, which translated into an increase of Bmp4 expression in STO feeder cells. Similarly, down-regulation of Bmp4 by activation of the NF-KB signaling pathway was found in other kinds of cells (Muraoka et al., 2000; Zhu et al., 2007). This suggests that production of BMP4 in feeder cells is possibly induced by inhibition of the NF-kB pathway.

The major effect of BMP4 on the self-renewal of ESCs is accomplished by means of the inhibition of both extracellular receptor kinase (ERK) and p38 mitogen-activated protein kinase (MAPK) pathways (Qi et al., 2004). Suppression of the MAPK pathway by BMP4 maintains pluripotency of ES cells by regulating gene expression of the ESC markers, Oct4 and Nanog. In our study, enhanced levels of BMP4 in STO cells inhibited colony formation and increased expression of Oct4 and Nanog genes of mESCs in an undifferentiated culture system, whereas id gene expression was unchanged in mESCs. Since a critical level of Oct4 is known to be essential to maintain pluripotency of ESCs (Niwa et al., 2000), induced expression of Oct4 in our system may have contributed to inhibition of ESC self-renewal. Addition of exogenous BMP4 also decreased the colony number of undifferentiated mESCs in a dose-dependent manner. When $\mathrm{CHO}$ 
cells overexpressing BMP4 were implanted in the avascular region of quail embryos, endothelial growth and capillary plexus formation were enhanced (Reese et al., 2004). Our data is consistent with that of a recent study showing a significant increase in the proliferation rate after addition of a small amount of BMP4 to bone marrow stem cells but a decrease to the lowest rate after adding a larger amount of BMP4 (Mazaheri et al., 2011). Other reports also demonstrated that high doses significantly increased apoptosis and drastically reduced cell proliferation, whereas low doses of BMP4 significantly increased cultured cell content, reduced the number of apoptotic cells, and increased the number of cycling cells (Vicente Lopez et al., 2011). Taken together, these results showed that excess BMP4 produced in STO cells inhibited proliferation of ESCs. Because of a smaller change in the expression of BMP4 target genes (ids), it is unlikely that a single signaling pathway affected by BMP4 activation is capable of entirely inhibiting self-renewal of mESCs.

\section{Methods}

\section{STO feeder cells and mESC growth}

STO feeder cells (ATCC, Toronto Ontario, Canada) were plated in Dulbecco's Modified Eagle Medium (DMEM; Invitrogen, Carlsbad, CA) supplemented with $10 \%$ fetal bovine serum (FBS; WelGENE, Daegu, Korea), $100 \mathrm{U} / \mathrm{ml}$ penicillin, and $100 \mathrm{mg} / \mathrm{ml}$ streptomycin. Murine embryonic stem D3 cells (mESCs) were maintained on mitomycin C-pretreated STO feeder cells in DMEM supplemented with 15\% FBS, 1\% nonessential amino acid (Sigma, St. Louis, MO), $0.1 \mathrm{mM} \beta$-mercaptoethanol (Sigma), 1\% penicillin and streptomycin, and $1 \times 10^{6} \mathrm{U}$ LIF (Chemicon, Temecula, CA). The ESCs were passaged every three days to maintain their undifferentiated state.

\section{Infection of STO feeder cells with adenovirus}

The adenovirus vector containing dominant-negative $I_{\kappa} B$ (rAd-dnlkB) or control adenovirus containing green fluorescence protein ( $r A d-G F P)$ was propagated in 293 cells and purified by $\mathrm{CsCl}$ density gradient. The titer of adenovirus stock was determined as plaque forming units $(\mathrm{pfu}) / \mathrm{ml}$ by the limiting dilution assay. The viral preparations were dialyzed and stored at $-80^{\circ} \mathrm{C}$ until use. Post-confluent STO cells cultured in tissue culture dishes were washed once with complete PBS and incubated with the adenovirus in DMEM $(100 \mu \mathrm{l} /$ well $)$ at $37^{\circ} \mathrm{C}$ at different multiplicities of infection (MOI), that is, virus to cell ratio, as indicated. Fresh DMEM growth medium supplemented with $15 \%$ FBS was added after $1.5 \mathrm{~h}$, and cells were maintained for two more days. The adenovirus was washed off with PBS, and ESCs $\left(2 \times 10^{4}\right.$ cells $\left./ \mathrm{ml}\right)$ were seeded into these STO feeder cell dishes and cultured until assayed.

\section{[ $\left.{ }^{3} \mathrm{H}\right]$ Thymidine incorporation assay}

ESCs $\left(1 \times 10^{4}\right.$ cells $\left./ \mathrm{ml}\right)$ cultured on the STO feeder cells were transferred into 96-well plates and cultured for $24 \mathrm{~h}$. For cell proliferation assays, $\left[{ }^{3} \mathrm{H}\right.$ ] thymidine (Amersham Biosciences KOREA Ltd, Seoul, Korea) was added into the cell culture plates for $96 \mathrm{~h}$, and cells were harvested. Thymidine incorporation was determined in a scintillation counter.

\section{mESC culture in the STO-conditioned medium}

The STO feeder cells infected by adenovirus were cultured in T-flasks containing DMEM supplemented with $10 \%$ FBS. After culture for $24 \mathrm{~h}$, STO-conditioned media were prepared by collecting the supernatant from the STO cell culture medium. We centrifuged the supernatant at 1,200 rpm for $5 \mathrm{~min}$ followed by filtration through a Minisart filter (pore size $0.2 \mu \mathrm{m}$; Sigma). Filtered supernatant was used as the conditioned medium after supplementing with glucose, nonessential amino acid, $\beta$-mercaptoethanol, and antibiotics to the same concentrations as those in the ESC growth medium described above. Mouse ESCs $\left(1 \times 10^{4}\right.$ cells $/ \mathrm{ml}$ ) were seeded and cultured in Matrigel (BD BioSciences, Mountain View, CA)-coated cell culture plates at $37^{\circ} \mathrm{C}$ until observation by microscope. Exogenous BMP4 (R\&D Systems Inc., Minneapolis, MN) was added into the mESC culture medium once a day.

\section{RNA purification and RT-PCR}

STO cells were cultured to $90-95 \%$ confluence in STO medium and then in ES cell medium for $24 \mathrm{~h}$ before total RNA purification using TRI reagent (Sigma). cDNA was synthesized using the Superscript II first-strand synthesis kit (Invitrogen). PCR amplification was carried out with primers specific to mouse BMP4, Wnt3, Wnt5a, STAT3, LIF, Oct4, Nanog, Id1, Id2, Id3, and Id4. RT-PCR for GAPDH was used as an internal control in each experiment.

\section{Real time PCR}

Real time PCR was performed using the StepOnePlus ${ }^{\mathrm{TM}}$ Real-time PCR System (Applied Biosystems Inc., Foster city, CA) using Eva Green dye. The mRNA expression levels of BMP4 in Ad-dnlкB cells were compared to the expression levels in Ad-GFP cells. The levels of PCR product were calculated from standard curves established for each primer pair (Livak and Schmittgen, 2001).

\section{Supplemental data}

Supplemental data include a figure and can be found with this article online at http://e-emm.or.kr/article/article_files/ SP-44-7-06.pdf.

\section{Acknowledgements}

This work was supported by Priority Research Center Program (2011-0030746) and Basic Research Lab (BRL2011-0001570) through the National Research Foundation of Korea (NRF) funded by the Ministry of Education, 
Science, and Technology. K-H. Kim and H-I. Choi are supported by the BK-21 Fund.

\section{References}

Ahn JY, Park G, Shim JS, Lee JW, Oh IH. Intramarrow injection of beta-catenin-activated, but not naive mesenchymal stromal cells stimulates self-renewal of hematopoietic stem cells in bone marrow. Exp Mol Med 2010;42: 122-31

Bhardwaj G, Murdoch B, Wu D, Baker DP, Williams KP, Chadwick K, Ling LE, Karanu FN, Bhatia M. Sonic hedgehog induces the proliferation of primitive human hematopoietic cells via BMP regulation. Nat Immunol 2001;2:172-80

Bhatia M, Bonnet D, Kapp U, Wang JC, Murdoch B, Dick JE. Quantitative analysis reveals expansion of human hematopoietic repopulating cells after short-term ex vivo culture. J Exp Med 1997;186:619-24

Bhatia M, Bonnet D, Wu D, Murdoch B, Wrana J, Gallacher $\mathrm{L}$, Dick JE. Bone morphogenetic proteins regulate the developmental program of human hematopoietic stem cells. J Exp Med 1999;189:1139-48

Brons IG, Smithers LE, Trotter MW, Rugg-Gunn P, Sun B, Chuva de Sousa Lopes SM, Howlett SK, Clarkson A, Ahrlund-Richter L, Pedersen RA, Vallier L. Derivation of pluripotent epiblast stem cells from mammalian embryos. Nature 2007;448:191-5

De Robertis EM, Sasai Y. A common plan for dorsoventral patterning in Bilateria. Nature 1996;380:37-40

Fainsod A, Steinbeisser H, De Robertis EM. On the function of BMP-4 in patterning the marginal zone of the Xenopus embryo. EMBO J 1994;13:5015-25

Hao J, Li TG, Qi X, Zhao DF, Zhao GQ. WNT/beta-catenin pathway up-regulates Stat3 and converges on LIF to prevent differentiation of mouse embryonic stem cells. Dev Biol 2006; 290:81-91

Hollnagel A, OehImann V, Heymer J, Ruther U, Nordheim A. Id genes are direct targets of bone morphogenetic protein induction in embryonic stem cells. J Biol Chem 1999;274: 19838-45

Jones CM, Lyons KM, Hogan BL. Involvement of Bone Morphogenetic Protein-4 (BMP-4) and Vgr-1 in morphogenesis and neurogenesis in the mouse. Development 1991;111:531-42

Livak KJ, Schmittgen TD. Analysis of relative gene expression data using real-time quantitative PCR and the 2(-Delta Delta C(T)) Method. Methods 2001;25:402-8

Mazaheri Z, Movahedin M, Rahbarizadeh F, Amanpour S. Different doses of bone morphogenetic protein 4 promote the expression of early germ cell-specific gene in bone marrow mesenchymal stem cells. in vitro Cell Dev Biol Anim 2011;47: $521-5$

Muraoka RS, Bushdid PB, Brantley DM, Yull FE, Kerr LD. Mesenchymal expression of nuclear factor-kappaB inhibits epithelial growth and branching in the embryonic chick lung.
Dev Biol 2000;225:322-38

Murdoch B, Gallacher L, Chadwick K, Fellows F, Bhatia M. Human embryonic-derived hematopoietic repopulating cells require distinct factors to sustain in vivo repopulating function. Exp Hematol 2002;30:598-605

Niwa H, Miyazaki J, Smith AG. Quantitative expression of Oct-3/4 defines differentiation, dedifferentiation or selfrenewal of ES cells. Nat Genet 2000;24:372-6

Park YB, Kim YY, Oh SK, Chung SG, Ku SY, Kim SH, Choi YM, Moon SY. Alterations of proliferative and differentiation potentials of human embryonic stem cells during long-term culture. Exp Mol Med 2008;40:98-108

Pearson S, Sroczynska P, Lacaud G, Kouskoff V. The stepwise specification of embryonic stem cells to hematopoietic fate is driven by sequential exposure to Bmp4, activin A, bFGF and VEGF. Development 2008;135:1525-35

Qi X, Li TG, Hao J, Hu J, Wang J, Simmons H, Miura S, Mishina Y, Zhao GQ. BMP4 supports self-renewal of embryonic stem cells by inhibiting mitogen-activated protein kinase pathways. Proc Natl Acad Sci USA 2004;101: 6027-32

Reese DE, Hall CE, Mikawa T. Negative regulation of midline vascular development by the notochord. Dev Cell 2004;6: 699-708

Schenke-Layland K, Angelis E, Rhodes KE, HeydarkhanHagvall S, Mikkola HK, Maclellan WR. Collagen IV induces trophoectoderm differentiation of mouse embryonic stem cells. Stem Cells 2007;25:1529-38

Schulz TC, Noggle SA, Palmarini GM, Weiler DA, Lyons IG, Pensa KA, Meedeniya AC, Davidson BP, Lambert NA, Condie BG. Differentiation of human embryonic stem cells to dopaminergic neurons in serum-free suspension culture. Stem Cells 2004;22:1218-38

Smith AG, Heath JK, Donaldson DD, Wong GG, Moreau J, Stahl M, Rogers D. Inhibition of pluripotential embryonic stem cell differentiation by purified polypeptides. Nature 1988;336:688-90

Smith AG. Embryo-derived stem cells: of mice and men. Annu Rev Cell Dev Biol 2001;17:435-62

Vainio S, Karavanova I, Jowett A, Thesleff I. Identification of BMP-4 as a signal mediating secondary induction between epithelial and mesenchymal tissues during early tooth development. Cell 1993;75:45-58

Vicente Lopez MA, Vazquez Garcia MN, Entrena A, Olmedillas Lopez S, Garcia-Arranz M, Garcia-Olmo D, Zapata A. Low doses of bone morphogenetic protein 4 increase the survival of human adipose-derived stem cells maintaining their stemness and multipotency. Stem Cells Dev 2011;20:1011-9

Xu RH, Chen X, Li DS, Li R, Addicks GC, Glennon C, Zwaka TP, Thomson JA. BMP4 initiates human embryonic stem cell differentiation to trophoblast. Nat Biotechnol 2002;20: $1261-4$

Xu RH, Peck RM, Li DS, Feng X, Ludwig T, Thomson JA. Basic FGF and suppression of BMP signaling sustain undifferentiated proliferation of human ES cells. Nat Methods 
2005;2:185-90

Ying QL, Nichols J, Chambers I, Smith A. BMP induction of Id proteins suppresses differentiation and sustains embryonic stem cell self-renewal in collaboration with STAT3. Cell 2003;115:281-92
Zhu NL, Li C, Huang HH, Sebald M, Londhe VA, Heisterkamp $\mathrm{N}$, Warburton D, Bellusci S, Minoo P. TNF-alpha represses transcription of human Bone Morphogenetic Protein-4 in lung epithelial cells. Gene 2007;393:70-80 